served in this case, especially clearly when cells obtained from 8 days old culture were used which had lost the ability to interconvert sorbose and fructose substantially.

It is of utmost interest that the interconversion of sorbose and fructose is a nonepimeric isomerization of sugars at C-5. A non-epimeric isomerization at $\mathbf{C}-4$ of uronic acids by Serratia was reported by Payne ${ }^{4}$, but this is the first report on the non-epimeric

4) W.J. Payne, J. Bact., 75, 240 (1958). isomerization of ketoses by microorganisms.

These studies are still in progress now, and will be published in detail elsewhere.

\section{Osamu Terada \\ Shizuko Suzuki \\ Shukuo Kinoshita}

Tokyo Research Laboratory

Kyowa Hakko Kogyo Co., Ltd.

Machida, Tokyo

Received August 10, 1961

[Agr. Biol. Chem., Vol. 25, No. 11, p. 871 872, 1961]

\title{
Occurrence of 5-Ketofructose during Kojic Acid Formation from Sorbose by Acetobacter Species
}

Sir:

It was previously reported that kojic acid and other two $\gamma$-pyrones were formed from fructose through 5-ketofructose by Gluconobacter cerinus and related Acetobacter species ${ }^{1)}$.

It was reported by other workers ${ }^{2)}$ that kojic acid was also formed from sorbose by particular cultures of Acetobacter, but no intermediate or mechanism of its formation has yet been reported.

In attempts to investigate the mechanism of kojic acid formation from sorbose by Acetobacter suboxydans var. nonacetum IFO 3254, one of the particular cultures mentioned above, 5-ketofructose was detected in culture media together with three $\gamma$-pyrones, fructose

1) O. Terada, S. Suzuki and S. Kinoshita, J. Agr. Chem. Soc. Japan. in press.

2) K. Kondo and S. Wada, J. Ferm, Technol. (Japan), 27, 331 (1949). and residual sorbose.

Sugar and $\gamma$-pyrones were identified paper chromatographically, and 5-ketofructose was isolated as its bis-phenylhydrazone and identified with its elemental analysis, shown below, and identities of melting point $\left(144 \sim 146^{\circ} \mathrm{C}\right)$ and IR absorption spectrum (Fig. 1) with that of authentic specimen. Anal. Found: C, 60.21; H, 6.12; N, 15.28. Calcd. for $\mathrm{C}_{18} \mathrm{H}_{22} \mathrm{~N}_{4} \mathrm{O}_{4}$ : C, 60.32; $\mathrm{H}, 6.19 ; \mathrm{N}, 15.63 \%$.

The formation of kojic acid and other two $\gamma$-pyrones from 5-ketofructose was also proved by cultivation experiments using 5-ketofructose as the carbon source.

These results indicate that 5-ketofructose is a precursor of kojic acid and other $\gamma$-pyrones also in this case, as in the case of kojic acid formation from fructose by Gluconobacter cerinus, reported previously.

It is not clear whether the formation of 


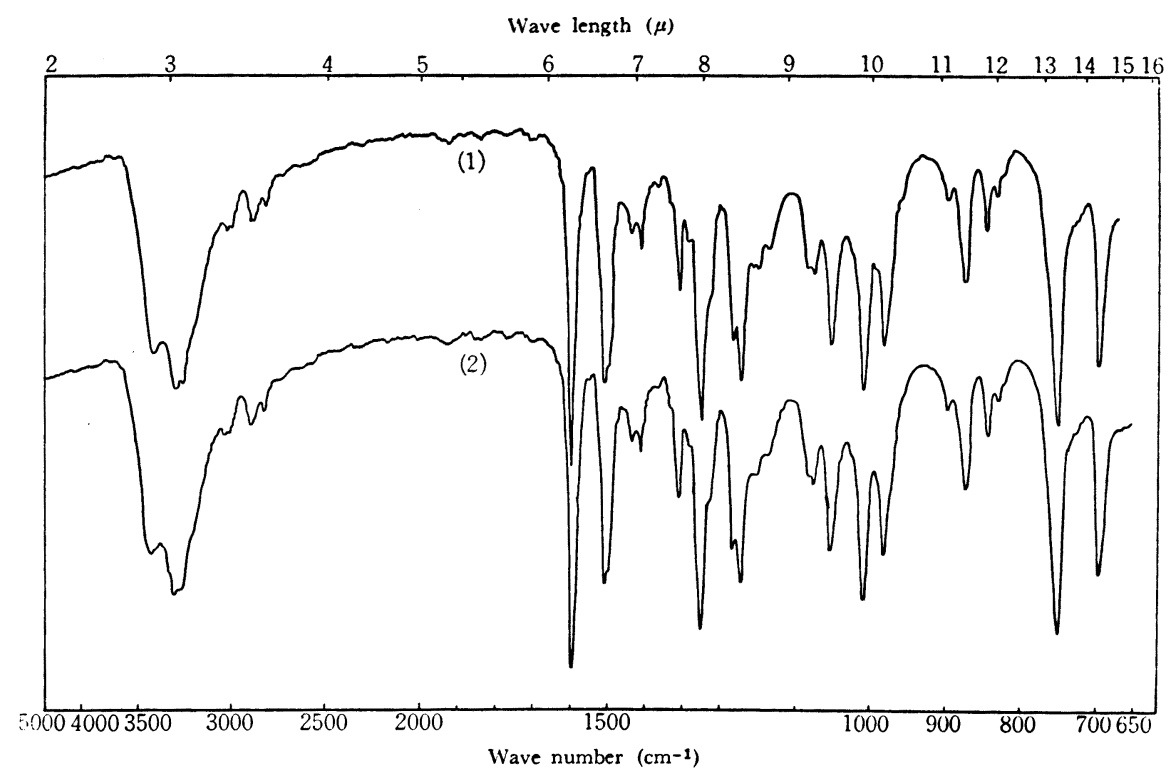

FIG. 1. IR-Absorption Spectra of Bis-phenylhydrazones.

(1) 5-Ketofructose-bis-phenylhydrazone (authentic)

(2) Sample

5-ketofructose from sorbose might proceed either directly from sorbose or via fructose after isomerization, but results obtained so far suggest the existence of both dehydrogenation, the former being stronger than the latter.

From these results, the authors would like to propose a possible pathway of kojic acid formation from sorbose as follows:

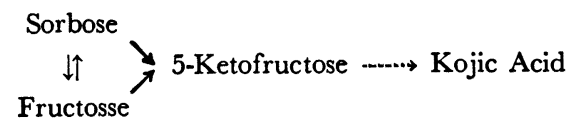

These studies are now in progress, and will be published elsewhere in detail.

\section{Osamu Terada \\ Shizuko Suzuki \\ Shukuo Kinoshita}

Tokyo Research Laboratory

Kyowa Hakko Kogyo Co., Ltd.

Machida, Tokyo 